\title{
Socioeconomic inequality in the prevalence of noncommunicable diseases in low- and middle-income countries: Results from the World Health Survey
}

\author{
Ahmad Reza Hosseinpoor ${ }^{1 *}$, Nicole Bergen ${ }^{1}$, Shanthi Mendis², Sam Harper ${ }^{3}$, Emese Verdes $^{1}$, Anton Kunst ${ }^{4}$ \\ and Somnath Chatterji ${ }^{1}$
}

\begin{abstract}
Background: Noncommunicable diseases are an increasing health concern worldwide, but particularly in low- and middle-income countries. This study quantified and compared education- and wealth-based inequalities in the prevalence of five noncommunicable diseases (angina, arthritis, asthma, depression and diabetes) and comorbidity in low- and middle-income country groups.
\end{abstract}

Methods: Using 2002-04 World Health Survey data from 41 low- and middle-income countries, the prevalence estimates of angina, arthritis, asthma, depression, diabetes and comorbidity in adults aged 18 years or above are presented for wealth quintiles and five education levels, by sex and country income group. Symptom-based classification was used to determine angina, arthritis, asthma and depression rates, and diabetes diagnoses were self-reported. Socioeconomic inequalities according to wealth and education were measured absolutely, using the slope index of inequality, and relatively, using the relative index of inequality.

Results: Wealth and education inequalities were more pronounced in the low-income country group than the middle-income country group. Both wealth and education were inversely associated with angina, arthritis, asthma, depression and comorbidity prevalence, with strongest inequalities reported for angina, asthma and comorbidity. Diabetes prevalence was positively associated with wealth and, to a lesser extent, education. Adjustments for confounding variables tended to decrease the magnitude of the inequality.

Conclusions: Noncommunicable diseases are not necessarily diseases of the wealthy, and showed unequal distribution across socioeconomic groups in low- and middle-income country groups. Disaggregated research is warranted to assess the impact of individual noncommunicable diseases according to socioeconomic indicators.

\section{Background}

The attenuation of the noncommunicable disease (NCD) burden has been cited as one of the greatest development challenges of the $21^{\text {st }}$ century $[1,2]$. In $2008,80 \%$ of the world's 36 million NCD-related deaths occurred in low- and middle-income countries (LMICs), which are home to most of the world's population [3]. Globally, cardiovascular disease and diabetes are responsible for

\footnotetext{
*Correspondence: hosseinpoora@who.int

1 Department of Health Statistics and Information Systems, World Health Organization, Geneva, Switzerland

Full list of author information is available at the end of the article
}

170 million disability adjusted life years $(11.3 \%$ of global burden), while cancers account for 78 million disability adjusted life years (5.1\% of global burden), and respiratory diseases, 60 million disability adjusted life years $(3.9 \%$ of global burden) [3]. Non-fatal NCDs, including depression [4] and arthritis [5] also contribute significantly to the global NCD burden. Without immediate and effective action, global NCD-related mortality is expected to reach 44 million in 2020, with a growing impact in lowresource settings [3].

NCDs are unequally distributed within populations, often disproportionally affecting the socioeconomically 
disadvantaged [3,6-9]. For example, a study of 52 countries at all stages of development found a trend for increased angina in poorer populations, although associations with individual- or societal-level socioeconomic markers were not analyzed [10]. A literature review reported elevated angina-related mortality and morbidity in less-affluent neighbourhoods, based on literature from several world regions [11]. Although few multinational studies of LMICs have examined the role of socioeconomic inequality in arthritis prevalence, preliminary research from the United States supported an association of low individual socioeconomic status with greater likelihood of arthritis in less-developed settings, and a potential role for community social determinants [12]. A lower level of formal education has been linked to higher arthritis prevalence [13-16], and worse arthritis outcomes [17] in high-income country settings. Studies that have analyzed associations between rates of asthma symptoms and country-level economic status have reported mixed findings [18-21], although greater asthma severity has been documented in populations of low socioeconomic status [20-22]. International depression studies universally report greater risk among women and populations of low economic standing or low levels of education [23,24], while diabetes was associated with low socioeconomic status in two high-income countries [25,26] and lower levels of education in middle- and high-income countries $[8,27,28]$. Previous studies from Southeast Asia reported associations between low education level and increased probability of having a chronic disease [29] and elevated number of chronic conditions [30].

In 2011, the international community convened at the United Nations High Level Summit to discuss approaches to address the rising trend of NCDs and their risk factors. In the absence of a concerted commitment amongst stakeholders, the international response thus far to alleviate this crisis has been deemed inadequate [31,32]. The amount of research dedicated to LMICs does not match the distribution of disease burden [33,34], and previous studies provide only a highly fragmented overview of the situation. Furthermore, prevention and treatment initiatives in lowresource settings are hindered by a lack of attention to social and economic situations [35].

The 2002-04 World Health Survey (WHS) gathered data about the symptoms of four major NCDs: angina, arthritis, asthma and depression as well as the self-reported diagnosis of diabetes. The objective of this study was to quantify and compare the socioeconomic inequalities in prevalence estimates of these five NCDs and comorbidity in LMICs.

\section{Methods}

\section{Study population}

Data were obtained from the 2002-04 WHS, conducted by the World Health Organization [36]. The WHS is a source of comparable population health data of adults aged 18 years and older in 70 countries from all regions of the world [37]. Survey samples were probabilistically selected, with all individuals having a known, non-zero chance of selection. WHS country surveys were nationally representative except in China, Comoros, Congo, Côte d'Ivoire, India, and the Russian Federation, where the WHS was carried out in geographically limited regions. To adjust for non-response and population distribution (as represented by the United Nations Statistical Division [38]), post-stratification corrections were made to sampling weights [4]. Informed consent was obtained in all surveys, using a procedure approved by institutional review boards [39]. The full list of local review boards from each study country is available in Additional file 1.

\section{Data}

This study focused on LMICs, classified according to the World Bank's development categories in 2003 [40], consistent with the timing of the majority of the WHS surveys. The 2002-04 WHS included 50 LMICs. Initially we assessed 48 LMICs that had available NCD prevalence data, and relevant socioeconomic and demographic data. (Guatemala did not have data on survey sampling weight, and Turkey had insufficient data to create the household wealth index, one of the principal variables of the study.) Seven countries were excluded from analysis because item non-response for any set of the questions pertaining to NCDs was over $20 \%$. The final sample comprised 170,298 respondents $(77,517$ men and 92,781 women) from 41 LMICs. Household level response rates were over $70 \%$ in all study countries except Czech Republic. Individual level response rates were above $82 \%$. See Additional file 2 for study country sample sizes, by sex, and Additional file 3, for NCD item non-response rates, by sex.

\section{Variables}

We analyzed data for angina, arthritis, asthma, depression, and diabetes. These represent five of the six chronic conditions included in section 6000 of the WHS individual questionnaire, and prominent NCDs worldwide. (Psychosis/schizophrenia was not included as a study variable because its prevalence was too low to allow for meaningful disaggregated analysis by SES indicators, controlling for potential confounders.) Symptom-based classification was used to determine angina, arthritis, asthma and depression rates, and diabetes diagnoses were self-reported. The diagnostic criteria to estimate the prevalence of each disease has been previously described [4]. Briefly, angina was diagnosed based on an algorithm derived from the Rose questionnaire [41]. Arthritis and asthma diagnoses were based on responses to validated 
symptom-related questions [42-44]. Depression diagnosis was based on International Classification of Disease tenth revision: Diagnosis Criteria for Research for Depressive Episodes [45], and was derived from an algorithm that took into account depression symptoms $[4,46]$. For each diagnosis all potential answer combinations were considered, and the best result based on Receiver Operator Characteristic analysis within each country was applied as diagnosis criteria [47]. Diagnoses of these diseases were based on questions about the respondent's condition in the 12 months preceding the interview date. For diabetes, diagnosis was based on a self-reported previous diagnosis. Comorbidity was defined as reporting of two or more NCDs by one individual. Because diabetes diagnosis was based solely on self-reported diagnosis (whereas symptom questions and the algorithm approach were used to diagnose the other NCDs), an additional comorbidity measure excluding diabetes was constructed.

Socioeconomic status was derived from household wealth status and individual highest-attained level of education. To measure household wealth, a dichotomous hierarchical ordered probit model was used to develop an index of the long-running economic status of households based on owning selected assets and/or using certain services [48-50]. The index was then divided into five quintiles within each country, with quintile one representing the poorest wealth quintile and quintile five, the richest. Education was ranked according to five categories: no formal schooling, less than primary school, primary school completed, secondary/high school completed, and college completed or above.

Confounders included sex, age (expressed categorically as $18-29,30-39,40-49,50-59,60-69$ and 70 or more years), marital status (married/cohabiting, divorced/separated/ widowed, or never married), area of residence (rural or urban), and country of residence.

\section{Methods of analysis}

Prevalence rates for each NCD and comorbidity were calculated for men and women in LIC and MIC groups, according to wealth quintiles and education level. Both age-standardized [51] and crude prevalence estimates were calculated.

Socioeconomic inequality in each NCD and comorbidity prevalence was measured using the slope index of inequality (SII) and the relative index of inequality (RII), measures that take into account the distribution of the population across wealth quintiles or education levels [52]. Poisson regression model with a robust variance was used to assess the association between each NCD prevalence and socioeconomic status and to generate prevalence difference and prevalence ratio estimates, and 95\% confidence intervals (95\%CI) [53]. To calculate SII and RII, individuals were cumulatively ranked (ranging from zero to one) according to descending socioeconomic status (i.e. highest wealth or education level to lowest). The exposure variable can thus be interpreted as a continuous measure, with a value of zero equivalent to the top of the socioeconomic distribution and a value of one equivalent to the bottom. Therefore, SII is the prevalence rate difference--and RII, the prevalence rate ratio-- between those at top rank (representing the lowest level of wealth or education) and those at rank zero (representing the highest level of wealth or education). A SII value greater than zero and a RII value greater than one indicated an inverse gradient, where NCD prevalence was greater among populations of lower socioeconomic status. We referred to this situation as "regular" inequality, and conversely, "reverse" inequality if prevalence was higher among those with higher socioeconomic position [54]. Data were adjusted for country of residence and age (Model 1), as well as other confounding factors: marital status, urban/rural area and education or wealth (Model 2).

Stata ${ }^{\circledR} 11$ was used for all analyses. We imputed missing values five times using Multiple Imputation by Integrated Chained Equations Technique [55]. All analyses were weighted, accounting for individual survey sample designs. The non-independence of observations within the surveys clusters were also incorporated in the analysis.

\section{Results}

Table 1 shows overall age-standardized prevalence of NCDs and comorbidity in men and women living in study LMICs. Overall, angina showed the highest prevalence rate. Overall age-standardized prevalence tended to be elevated in women. Except for depression, agestandardized prevalence rates were higher in MICs. Crude prevalence rates of NCDs and comorbidity are shown in Additional file 4.

\section{Wealth-related inequality}

Table 2 summarizes age-standardized prevalence rates of NCDs and comorbidity by household wealth quintile and absolute inequalities (SII), among men and women living in study LMICs. Relative inequalities are illustrated in Figure 1, and RII values are provided in Additional file 5. Additional file 6 summarizes crude prevalence of NCDs by wealth among each sexincome group.

We generally found regular inequality in prevalence values of angina, arthritis, asthma and depression in both absolute and relative terms, after controlling for respondents' age and country of residence (Model 1). Of all NCDs, angina consistently demonstrated the highest absolute inequality. Notably, the absolute difference of angina prevalence between poorest and richest women 
Table 1 Age-standardized prevalence (\%) of noncommunicable diseases among adults of 41 low- and middle-income countries, World Health Survey 2002-04

\begin{tabular}{|c|c|c|c|c|c|c|c|c|c|c|c|c|}
\hline \multirow[b]{4}{*}{ Angina } & \multicolumn{6}{|c|}{ Men } & \multicolumn{6}{|c|}{ Women } \\
\hline & \multicolumn{3}{|c|}{ Middle-income group } & \multicolumn{3}{|c|}{ Low-income group } & \multicolumn{3}{|c|}{ Middle-income group } & \multicolumn{3}{|c|}{ Low-income group } \\
\hline & \multirow{2}{*}{$\begin{array}{c}\text { Estimate } \\
9.8\end{array}$} & \multicolumn{2}{|c|}{$95 \% \mathrm{Cl}$} & \multirow{2}{*}{$\begin{array}{c}\text { Estimate } \\
9.1\end{array}$} & \multicolumn{2}{|c|}{$95 \% \mathrm{Cl}$} & \multirow{2}{*}{$\begin{array}{c}\text { Estimate } \\
15.1\end{array}$} & \multicolumn{2}{|c|}{$95 \% \mathrm{Cl}$} & \multirow{2}{*}{$\begin{array}{c}\text { Estimate } \\
14.1\end{array}$} & \multicolumn{2}{|c|}{$95 \% \mathrm{Cl}$} \\
\hline & & 9.2 & 10.3 & & 8.6 & 9.5 & & 14.4 & 15.7 & & 13.6 & 14.6 \\
\hline Arthritis & 6.6 & 6.2 & 7.1 & 4.4 & 4.1 & 4.7 & 10.0 & 9.5 & 10.4 & 6.1 & 5.7 & 6.4 \\
\hline Asthma & 6.4 & 6.0 & 6.8 & 5.5 & 5.1 & 5.9 & 6.8 & 6.4 & 7.2 & 6.4 & 6.0 & 6.8 \\
\hline Depression & 3.9 & 3.6 & 4.2 & 4.9 & 4.5 & 5.2 & 7.0 & 6.5 & 7.4 & 7.8 & 7.4 & 8.2 \\
\hline Diabetes & 3.7 & 3.3 & 4.0 & 1.4 & 1.2 & 1.6 & 5.0 & 4.7 & 5.3 & 1.5 & 1.3 & 1.7 \\
\hline Co-morbidity & 6.3 & 5.9 & 6.7 & 4.6 & 4.3 & 4.9 & 10.1 & 9.6 & 10.6 & 7.1 & 6.7 & 7.4 \\
\hline
\end{tabular}

of the MIC group was over $10 \%$ points (prevalence difference: $10.6 \%$, 95\%CI 7.2\%-13.9\%). Arthritis demonstrated the weakest inequality, and was non-significant after controlling for other study confounders (Model 2). Diabetes prevalence was positively associated with increasing wealth quintile. This reverse inequality was more pronounced in the LIC group, and lost significance in MICs when data were adjusted for confounders (Model 2). For example, diabetes prevalence in the poorest women living in the study LIC group was one fourth of the richest in Model 2 (prevalence ratio: 0.24, 95\% CI 0.14-0.44).

Comorbidity prevalence showed an inverse association with wealth quintile in all four sex-income groups; i.e. regular inequality. In both country income groups comorbidity was around 1.5 times more prevalent in the poorest women than in the richest in Model 1 (prevalence ratio: women of study LICs: 1.46, 95\%CI 1.19-1.80; women of study MICs: 1.51 , 95\%CI 1.14-1.98). Comorbidity was 2.5 times more prevalent in the poorest men of study LICs than in the richest (prevalence ratio: 2.50, 95\%CI 1.89-3.30). Wealth-related inequalities in comorbidity remained statistically significant in Model 2 in all sex-income groups except for women living in the LIC group. Excluding diabetes from the comorbidity measure strengthened regular inequality, which was robust to adjustment for confounding.

\section{Education-related inequality}

Table 3 summarizes age-standardized prevalence rates of NCDs and comorbidity by education and absolute inequalities (SII), among men and women living in study LMICs. Relative inequalities are illustrated in Figure 2, and RII values are provided in Additional file 7. Additional file 8 summarizes crude prevalence of NCDs by education among each sex-income group.

Controlling for age and country of residence, regular inequalities were reported for the prevalence of angina and asthma in most study populations. Education-related inequality was weak or non-significant for arthritis prevalence (except among men of LICs), and also for depression prevalence (except among women of LICs). Depression was twice as prevalent among LIC women with no formal education as women with college/university education (prevalence ratio: 2.19, 95\%CI 1.68-2.87). The strongest education-related regular inequality was found among LIC populations for angina. The absolute difference of angina prevalence between adults with no formal education and those with college/university education was near $8 \%$ points (prevalence difference: men: $7.6 \%$, 95\%CI 5.2\%-10.0\%; women: 7.8\%, 95\%CI 4.6\%-11.0\%). In MICs, all education-related inverse associations were non-significant after adjusting for other confounding factors (Model 2). Diabetes prevalence was associated with increasing education level (reverse inequality), demonstrating a significant relationship in LICs, after adjusting for all confounding factors (prevalence ratio: men: 0.36 , 95\%CI 0.20-0.64; women: 0.44 , 95\% CI 0.22 0.88).

Comorbidity showed regular inequality, with an inverse association between comorbidity prevalence and education level in all sex-income groups. The absolute difference in comorbidity prevalence among adults living in the LIC group was about 5\% points across educational levels in Model 1. Education-related inequality was robust to adjustment for confounding in the LIC group, but not the MIC group.

\section{Discussion}

Within LICs and MICs, wealth- and education-related inequalities of variable magnitudes and direction were quantified for the five NCDs. In most populations, regular inequalities in terms of wealth and education were reported for angina, arthritis, asthma and depression, with the strongest associations for angina, asthma and comorbidity. For all NCDs, additional adjustments for confounding factors in Model 2 (marital status, urban/ rural area and wealth/education) tended to decrease the magnitude of inequality, and may thus help to explain disparities in NCD prevalence. 
Table 2 Noncommunicable disease prevalence (\%) by wealth quintile, and wealth-related inequality among adults of 41 low- and middle-income countries, World Health Survey 2002-04

\begin{tabular}{|c|c|c|c|c|c|c|c|c|c|c|c|c|c|c|c|c|c|c|c|c|c|}
\hline \multirow[b]{3}{*}{ Men } & \multirow{4}{*}{$\begin{array}{l}\text { Middle-income } \\
\text { group }\end{array}$} & \multirow[b]{3}{*}{ Wealth quintile 1} & & \multicolumn{3}{|c|}{ Angina } & \multicolumn{3}{|c|}{ Arthritis } & \multicolumn{3}{|c|}{ Asthma } & \multicolumn{3}{|c|}{ Depression } & \multicolumn{3}{|c|}{ Diabetes } & \multicolumn{3}{|c|}{ Co-morbidity } \\
\hline & & & & \multirow{2}{*}{$\begin{array}{c}\text { Estimate } \\
13.0\end{array}$} & \multicolumn{2}{|c|}{$95 \% \mathrm{Cl}$} & \multirow{2}{*}{$\begin{array}{c}\text { Estimate } \\
6.9\end{array}$} & \multicolumn{2}{|c|}{$95 \% \mathrm{Cl}$} & \multirow{2}{*}{$\begin{array}{c}\text { Estimate } \\
9.5\end{array}$} & \multicolumn{2}{|c|}{$95 \% \mathrm{Cl}$} & \multirow{2}{*}{$\begin{array}{c}\text { Estimate } \\
5.5\end{array}$} & \multicolumn{2}{|c|}{$95 \% \mathrm{Cl}$} & \multirow{2}{*}{$\begin{array}{c}\text { Estimate } \\
2.7\end{array}$} & \multicolumn{2}{|c|}{$95 \% \mathrm{Cl}$} & \multirow{2}{*}{$\begin{array}{c}\text { Estimate } \\
8.3\end{array}$} & \multicolumn{2}{|c|}{$95 \% \mathrm{Cl}$} \\
\hline & & & & & 11.9 & 14.1 & & 6.1 & 7.7 & & 8.4 & 10.6 & & 4.7 & 6.2 & & 2.2 & 3.2 & & 7.4 & 9.1 \\
\hline & & Wealth quintile 2 & & 10.5 & 9.2 & 11.8 & 6.8 & 6.0 & 7.6 & 7.7 & 6.7 & 8.6 & 4.0 & 3.2 & 4.8 & 3.0 & 2.4 & 3.6 & 6.6 & 5.8 & 7.4 \\
\hline & & Wealth quintile 3 & & 9.8 & 8.8 & 10.8 & 6.5 & 5.8 & 7.2 & 6.6 & 5.8 & 7.4 & 3.9 & 3.3 & 4.6 & 4.1 & 3.5 & 4.7 & 6.3 & 5.5 & 7.0 \\
\hline & & Wealth quintile 4 & & 8.9 & 8.0 & 9.8 & 7.0 & 6.2 & 7.8 & 5.7 & 5.0 & 6.4 & 4.4 & 3.7 & 5.2 & 3.7 & 3.2 & 4.2 & 6.1 & 5.3 & 6.8 \\
\hline & & Wealth quintile 5 & & 6.9 & 6.0 & 7.8 & 5.1 & 4.3 & 5.8 & 4.1 & 3.5 & 4.7 & 2.3 & 1.8 & 2.8 & 4.3 & 3.8 & 4.9 & 4.1 & 3.4 & 4.8 \\
\hline & & \multirow{2}{*}{$\begin{array}{l}\text { Slope index } \\
\text { of inequality }\end{array}$} & Model 1* & 7.3 & 5.0 & 9.6 & 2.4 & 0.3 & 4.5 & 5.4 & 3.4 & 7.5 & 2.5 & 1.1 & 3.8 & -1.7 & -2.8 & -0.5 & 4.6 & 2.5 & 6.6 \\
\hline & & & Model $2^{* *}$ & 6.4 & 3.7 & 9.1 & 1.5 & -1.0 & 4.0 & 3.2 & 1.0 & 5.4 & 2.9 & 1.0 & 4.8 & -0.8 & -2.2 & 0.6 & 3.5 & 1.0 & 6.0 \\
\hline & \multirow{7}{*}{$\begin{array}{l}\text { Low-income } \\
\text { group }\end{array}$} & Wealth quintile 1 & & 10.8 & 9.9 & 11.6 & 4.5 & 3.9 & 5.1 & 6.7 & 5.9 & 7.5 & 5.7 & 5.0 & 6.4 & 0.7 & 0.4 & 1.0 & 5.7 & 5.1 & 6.4 \\
\hline & & Wealth quintile 2 & & 10.9 & 10.0 & 11.8 & 5.0 & 4.4 & 5.6 & 5.7 & 5.0 & 6.4 & 4.8 & 4.2 & 5.5 & 1.0 & 0.7 & 1.3 & 5.1 & 4.4 & 5.7 \\
\hline & & Wealth quintile 3 & & 9.3 & 8.4 & 10.2 & 4.2 & 3.7 & 4.7 & 5.9 & 5.2 & 6.7 & 4.8 & 4.1 & 5.4 & 1.2 & 0.9 & 1.4 & 4.9 & 4.2 & 5.5 \\
\hline & & Wealth quintile 4 & & 8.2 & 7.4 & 9.0 & 4.7 & 4.0 & 5.4 & 5.4 & 4.7 & 6.0 & 4.7 & 4.0 & 5.5 & 1.4 & 1.1 & 1.7 & 4.4 & 3.7 & 5.1 \\
\hline & & Wealth quintile 5 & & 6.6 & 5.7 & 7.4 & 3.7 & 3.2 & 4.3 & 4.4 & 3.8 & 5.0 & 4.1 & 3.5 & 4.7 & 2.5 & 2.1 & 2.9 & 3.3 & 2.7 & 3.9 \\
\hline & & Slope index & Model $1^{*}$ & 7.8 & 5.9 & 9.8 & 2.2 & 0.8 & 3.7 & 4.1 & 2.7 & 5.4 & 2.5 & 0.8 & 4.2 & -3.4 & -4.5 & -2.2 & 5.0 & 3.5 & 6.5 \\
\hline & & & Model $2^{* *}$ & 5.0 & 2.8 & 7.3 & 0.6 & -1.0 & 2.3 & 2.5 & 0.7 & 4.4 & 2.0 & 0.2 & 3.9 & -1.4 & -2.7 & -0.2 & 3.5 & 1.6 & 5.4 \\
\hline Women & Middle-income & Wealth quintile 1 & & 18.6 & 17.4 & 19.9 & 9.6 & 8.7 & 10.4 & 9.2 & 8.4 & 10.1 & 9.1 & 8.2 & 10.0 & 4.2 & 3.6 & 4.9 & 12.2 & 11.3 & 13.1 \\
\hline & & Wealth quintile 2 & & 16.9 & 15.7 & 18.1 & 10.5 & 9.6 & 11.4 & 7.8 & 6.9 & 8.6 & 7.3 & 6.6 & 8.1 & 4.9 & 4.3 & 5.5 & 11.4 & 10.4 & 12.4 \\
\hline & & Wealth quintile 3 & & 15.6 & 14.4 & 16.8 & 10.1 & 9.3 & 11.0 & 7.1 & 6.3 & 7.8 & 7.0 & 6.1 & 7.8 & 4.6 & 4.1 & 5.1 & 10.4 & 9.5 & 11.3 \\
\hline & & Wealth quintile 4 & & 13.4 & 12.3 & 14.5 & 10.3 & 9.4 & 11.1 & 5.4 & 4.8 & 5.9 & 7.5 & 6.7 & 8.3 & 5.2 & 4.5 & 5.8 & 9.5 & 8.7 & 10.4 \\
\hline & & Wealth quintile 5 & & 11.9 & 10.8 & 12.9 & 9.8 & 8.9 & 10.7 & 5.6 & 5.0 & 6.2 & 5.1 & 4.4 & 5.8 & 5.6 & 4.9 & 6.3 & 8.3 & 7.5 & 9.2 \\
\hline & & Slope index & Model $1^{*}$ & 10.6 & 7.2 & 13.9 & 1.0 & -1.5 & 3.5 & 4.9 & 1.7 & 8.2 & 3.7 & 1.9 & 5.5 & -2.2 & -4.9 & 0.5 & 4.8 & 1.7 & 7.8 \\
\hline & & & Model $2^{* *}$ & 10.4 & 6.7 & 14.2 & 1.2 & -1.8 & 4.3 & 4.4 & 0.9 & 8.0 & 3.6 & 1.4 & 5.8 & -2.5 & -5.7 & 0.7 & 4.3 & 0.8 & 7.9 \\
\hline & Low-income & Wealth quintile 1 & & 15.4 & 14.4 & 16.3 & 6.3 & 5.6 & 7.0 & 6.4 & 5.7 & 7.1 & 8.6 & 7.7 & 9.4 & 0.6 & 0.4 & 0.9 & 7.3 & 6.6 & 8.0 \\
\hline & & Wealth quintile 2 & & 15.7 & 14.7 & 16.8 & 6.6 & 5.9 & 7.2 & 7.4 & 6.6 & 8.2 & 8.5 & 7.7 & 9.3 & 1.0 & 0.7 & 1.2 & 7.9 & 7.2 & 8.7 \\
\hline & & Wealth quintile 3 & & 14.0 & 12.9 & 15.2 & 6.8 & 6.0 & 7.5 & 6.5 & 5.7 & 7.3 & 8.2 & 7.4 & 9.1 & 1.1 & 0.8 & 1.4 & 7.4 & 6.6 & 8.2 \\
\hline & & Wealth quintile 4 & & 13.2 & 12.1 & 14.3 & 5.8 & 5.1 & 6.4 & 5.8 & 5.1 & 6.5 & 7.6 & 6.8 & 8.4 & 1.7 & 1.3 & 2.0 & 6.4 & 5.7 & 7.1 \\
\hline & & Wealth quintile 5 & & 11.9 & 10.6 & 13.2 & 5.5 & 4.7 & 6.3 & 5.9 & 5.2 & 6.6 & 5.8 & 5.0 & 6.6 & 3.2 & 2.7 & 3.7 & 6.3 & 5.5 & 7.2 \\
\hline & & Slope index & Model $1^{*}$ & 7.0 & 4.3 & 9.7 & 2.0 & 0.3 & 3.6 & 1.1 & -0.4 & 2.6 & 4.2 & 2.3 & 6.2 & -4.0 & -5.3 & -2.8 & 3.1 & 1.4 & 4.8 \\
\hline & & of inequality & Model $2^{* *}$ & 4.9 & 1.1 & 8.7 & 1.1 & -1.1 & 3.2 & 0.5 & -1.3 & 2.2 & 1.8 & -0.7 & 4.3 & -2.7 & -3.9 & -1.6 & 1.2 & -0.9 & 3.3 \\
\hline
\end{tabular}




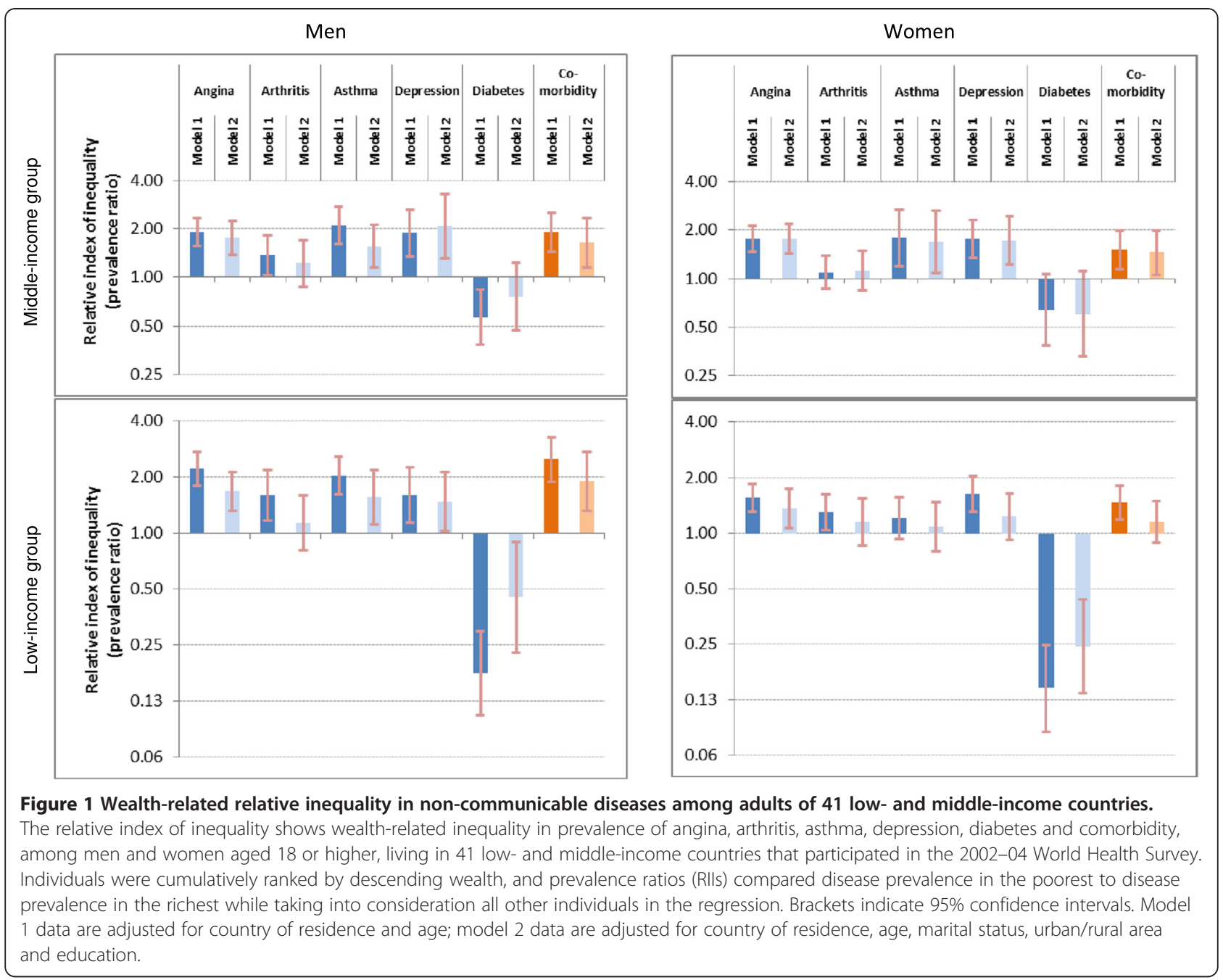

These findings are in accordance with previous reports from a variety of settings, which also reported inverse associations between socioeconomic position and prevalence of angina $[10,11,56]$, arthritis $[12,13,15,16]$, asthma [57,58], and depression [23,24,59-62]. Previously, European data (including eight higher-income countries) from the 1990s demonstrated educationrelated inequality in 14 of 17 studied NCDs, most notably stroke, diseases of the nervous system and diabetes. There was a tendency for stronger inequality in adults aged 25-59 than those aged 60-79 [9]. Goyal et al. (2010) highlighted differences based on country income group, reporting that education had more of a protective effect against cardiovascular events in high-income countries than LMICs, and also among men [27].

The connection between socioeconomic status and health is complex, and shaped by diverse circumstantial factors as well as political, social and economic forces [63]. For example, people living in poverty may experience material deprivation and high stress levels, which may lead to constrained choices and a higher likelihood of engaging in risky health behaviours, increasing the risk of disease; following disease onset, reduced access to care hinders opportunities to prevent complications [64]. It has been estimated that up to $80 \%$ of cases of cardiovascular disease or type 2 diabetes and $40 \%$ of cancer cases are preventable based on current knowledge, however, prevention initiatives may not adequately reach vulnerable populations where disease risk factors cluster [65].

Unlike the other four NCDs, we reported higher diabetes prevalence among the wealthier and more educated, especially in LICs. These findings conflict with trends reported by previous studies conducted in higherincome countries $[25,26]$. Epidemiological studies in lower-income countries are less-forthcoming, however diabetes was reported to be positively associated with affluence in the Dominican Republic [66], and metabolic syndrome was positively associated with affluence among 
Table 3 Noncommunicable disease prevalence (\%) by education level, and education-related inequality among adults of 41 low- and middle-income countries, World Health Survey 2002-04

\begin{tabular}{|c|c|c|c|c|c|c|c|c|c|c|c|c|c|c|c|c|c|c|c|c|c|}
\hline \multirow[b]{3}{*}{ Men } & \multirow[b]{3}{*}{$\begin{array}{l}\text { Middle-income } \\
\text { group }\end{array}$} & \multirow{3}{*}{\multicolumn{2}{|c|}{$\begin{array}{l}\text { No formal } \\
\text { schooling }\end{array}$}} & \multicolumn{3}{|c|}{ Angina } & \multicolumn{3}{|c|}{ Arthritis } & \multicolumn{3}{|c|}{ Asthma } & \multicolumn{3}{|c|}{ Depression } & \multicolumn{3}{|c|}{ Diabetes } & \multicolumn{3}{|c|}{ Co-morbidity } \\
\hline & & & & \multirow{2}{*}{$\begin{array}{c}\text { Estimate } \\
12.0\end{array}$} & \multicolumn{2}{|c|}{$95 \% \mathrm{Cl}$} & \multirow{2}{*}{$\begin{array}{c}\text { Estimate } \\
7.9\end{array}$} & \multicolumn{2}{|c|}{$95 \% \mathrm{Cl}$} & \multirow{2}{*}{$\begin{array}{c}\text { Estimate } \\
9.2\end{array}$} & \multicolumn{2}{|c|}{$95 \% \mathrm{Cl}$} & \multirow{2}{*}{$\begin{array}{c}\text { Estimate } \\
7.1\end{array}$} & \multicolumn{2}{|c|}{$95 \% \mathrm{Cl}$} & \multirow{2}{*}{$\begin{array}{c}\text { Estimate } \\
2.7\end{array}$} & \multicolumn{2}{|c|}{$95 \% \mathrm{Cl}$} & \multirow{2}{*}{$\begin{array}{c}\text { Estimate } \\
7.7\end{array}$} & \multicolumn{2}{|c|}{$95 \% \mathrm{Cl}$} \\
\hline & & & & & 10.4 & 13.7 & & 6.4 & 9.3 & & 7.7 & 10.8 & & 5.8 & 8.3 & & 1.9 & 3.4 & & 6.4 & 9.0 \\
\hline & & $\begin{array}{l}\text { Less than } \\
\text { primary schoo }\end{array}$ & & 10.5 & 9.3 & 11.8 & 7.9 & 6.7 & 9.0 & 6.8 & 5.9 & 7.8 & 5.2 & 4.2 & 6.1 & 3.7 & 3.0 & 4.4 & 7.8 & 6.7 & 9.0 \\
\hline & & $\begin{array}{l}\text { Primary schoo } \\
\text { completed }\end{array}$ & & 10.9 & 9.7 & 12.1 & 6.0 & 5.2 & 6.8 & 7.4 & 6.5 & 8.2 & 5.2 & 4.4 & 6.0 & 4.3 & 3.8 & 4.9 & 7.4 & 6.5 & 8.2 \\
\hline & & $\begin{array}{l}\text { Secondary/hig } \\
\text { completed }\end{array}$ & gh school & 8.5 & 7.8 & 9.2 & 6.1 & 5.5 & 6.7 & 5.7 & 5.1 & 6.3 & 3.2 & 2.7 & 3.7 & 4.1 & 3.6 & 4.7 & 5.2 & 4.7 & 5.8 \\
\hline & & $\begin{array}{l}\text { College comp } \\
\text { or above }\end{array}$ & bleted & 6.6 & 5.7 & 7.4 & 5.5 & 4.6 & 6.4 & 4.2 & 3.0 & 5.3 & 2.6 & 1.9 & 3.3 & 4.0 & 3.3 & 4.7 & 4.3 & 3.7 & 4.9 \\
\hline & & \multirow{2}{*}{$\begin{array}{l}\text { Slope index } \\
\text { of inequality }\end{array}$} & Model $1^{*}$ & 4.8 & 2.0 & 7.5 & 1.4 & -1.0 & 3.8 & 5.3 & 2.7 & 7.8 & 1.6 & -0.1 & 3.3 & -1.1 & -2.5 & 0.3 & 3.1 & 0.7 & 5.6 \\
\hline & & & Model $2^{* *}$ & 1.0 & -2.1 & 4.0 & -0.2 & -2.9 & 2.5 & 2.5 & -0.1 & 5.1 & 0.5 & -1.6 & 2.6 & 0.0 & -1.5 & 1.4 & 0.6 & -2.1 & 3.4 \\
\hline & \multirow[t]{7}{*}{$\begin{array}{l}\text { Low-income } \\
\text { group }\end{array}$} & \multicolumn{2}{|l|}{$\begin{array}{l}\text { No formal } \\
\text { schooling }\end{array}$} & 10.2 & 9.4 & 11.1 & 5.2 & 4.6 & 5.8 & 6.4 & 5.7 & 7.1 & 5.5 & 4.8 & 6.1 & 0.8 & 0.6 & 1.0 & 5.5 & 4.9 & 6.1 \\
\hline & & \multicolumn{2}{|l|}{$\begin{array}{l}\text { Less than } \\
\text { primary school }\end{array}$} & 10.1 & 9.1 & 11.1 & 4.2 & 3.6 & 4.7 & 5.7 & 4.8 & 6.5 & 4.9 & 4.2 & 5.7 & 1.4 & 1.0 & 1.8 & 5.1 & 4.4 & 5.8 \\
\hline & & \multicolumn{2}{|l|}{$\begin{array}{l}\text { Primary school } \\
\text { completed }\end{array}$} & 8.2 & 7.3 & 9.1 & 3.4 & 2.8 & 3.9 & 6.0 & 5.0 & 6.9 & 4.4 & 3.6 & 5.1 & 1.6 & 1.2 & 2.1 & 3.9 & 3.2 & 4.5 \\
\hline & & \multicolumn{2}{|c|}{$\begin{array}{l}\text { Secondary/high school } \\
\text { completed }\end{array}$} & 6.4 & 5.6 & 7.3 & 3.5 & 2.8 & 4.2 & 4.6 & 3.8 & 5.3 & 3.8 & 3.1 & 4.6 & 2.6 & 2.1 & 3.2 & 3.0 & 2.5 & 3.6 \\
\hline & & $\begin{array}{l}\text { College comp } \\
\text { or above }\end{array}$ & pleted & 4.4 & 3.2 & 5.7 & 3.0 & 2.1 & 4.0 & 3.7 & 2.8 & 4.7 & 4.1 & 3.0 & 5.1 & 2.8 & 2.0 & 3.5 & 2.5 & 1.7 & 3.3 \\
\hline & & Slope index & Model $1^{*}$ & 7.6 & 5.2 & 10.0 & 2.9 & 1.1 & 4.7 & 4.1 & 2.3 & 5.9 & 1.9 & -0.4 & 4.2 & -3.7 & -4.9 & -2.4 & 4.8 & 3.1 & 6.5 \\
\hline & & of inequality & Model $2^{* *}$ & 4.1 & 1.4 & 6.9 & 2.2 & 0.2 & 4.2 & 2.4 & 0.2 & 4.6 & 0.3 & -2.4 & 3.0 & -2.0 & -3.2 & -0.8 & 2.7 & 0.7 & 4.7 \\
\hline Women & $\begin{array}{l}\text { Middle-income } \\
\text { group }\end{array}$ & $\begin{array}{l}\text { No formal } \\
\text { schooling }\end{array}$ & & 17.9 & 16.4 & 19.4 & 10.7 & 9.6 & 11.8 & 10.2 & 9.0 & 11.4 & 12.0 & 10.5 & 13.5 & 7.7 & 6.7 & 8.7 & 15.2 & 13.9 & 16.5 \\
\hline & & $\begin{array}{l}\text { Less than } \\
\text { primary schoo }\end{array}$ & & 17.0 & 15.2 & 18.9 & 8.8 & 7.9 & 9.7 & 9.7 & 8.8 & 10.6 & 7.0 & 5.9 & 8.0 & 5.7 & 5.1 & 6.4 & 11.6 & 10.7 & 12.6 \\
\hline & & $\begin{array}{l}\text { Primary schoo } \\
\text { completed }\end{array}$ & & 16.2 & 14.8 & 17.5 & 11.8 & 10.7 & 12.9 & 7.7 & 6.7 & 8.6 & 7.6 & 6.2 & 8.9 & 6.3 & 5.6 & 7.0 & 11.9 & 10.7 & 13.1 \\
\hline & & $\begin{array}{l}\text { Secondary/hig } \\
\text { completed }\end{array}$ & gh school & 13.3 & 12.5 & 14.2 & 8.8 & 8.1 & 9.5 & 6.2 & 5.7 & 6.8 & 5.7 & 5.0 & 6.4 & 5.1 & 4.5 & 5.7 & 8.8 & 8.1 & 9.5 \\
\hline & & $\begin{array}{l}\text { College comp } \\
\text { or above }\end{array}$ & leted & 9.5 & 8.5 & 10.4 & 8.8 & 7.9 & 9.6 & 3.9 & 3.3 & 4.6 & 5.1 & 4.3 & 6.0 & 2.9 & 2.4 & 3.5 & 5.6 & 4.9 & 6.3 \\
\hline & & Slope index & Model $1^{*}$ & 5.6 & 1.9 & 9.3 & 0.0 & -2.6 & 2.6 & 4.7 & 1.1 & 8.4 & 3.0 & 0.5 & 5.5 & 0.3 & -2.0 & 2.6 & 3.8 & 0.5 & 7.2 \\
\hline & & & Model $2^{* *}$ & 0.9 & -2.7 & 4.6 & -0.7 & -3.5 & 2.0 & 2.9 & -0.4 & 6.2 & 1.4 & -1.3 & 4.1 & 1.7 & -0.1 & 3.6 & 1.9 & -1.2 & 5.1 \\
\hline
\end{tabular}


Table 3 Noncommunicable disease prevalence (\%) by education level, and education-related inequality among adults of 41 low- and middle-income countries, World Health Survey 2002-04 (Continued)

\begin{tabular}{|c|c|c|c|c|c|c|c|c|c|c|c|c|c|c|c|c|c|c|c|c|}
\hline \multirow[t]{7}{*}{$\begin{array}{l}\text { Low-income } \\
\text { group }\end{array}$} & \multicolumn{2}{|l|}{$\begin{array}{l}\text { No formal } \\
\text { schooling }\end{array}$} & 14.7 & 13.9 & 15.5 & 6.1 & 5.7 & 6.6 & 7.1 & 6.5 & 7.7 & 8.4 & 7.8 & 9.0 & 1.1 & 0.9 & 1.3 & 7.7 & 7.1 & 8.2 \\
\hline & \multicolumn{2}{|c|}{$\begin{array}{l}\text { Less than } \\
\text { primary school }\end{array}$} & 15.1 & 13.9 & 16.3 & 6.4 & 5.4 & 7.5 & 5.7 & 5.0 & 6.4 & 7.4 & 6.5 & 8.3 & 2.6 & 2.0 & 3.3 & 7.1 & 6.2 & 8.0 \\
\hline & \multicolumn{2}{|c|}{$\begin{array}{l}\text { Primary school } \\
\text { completed }\end{array}$} & 11.6 & 10.2 & 13.0 & 5.4 & 4.5 & 6.3 & 6.2 & 5.2 & 7.2 & 5.9 & 5.0 & 6.8 & 2.2 & 1.7 & 2.7 & 5.4 & 4.5 & 6.3 \\
\hline & \multicolumn{2}{|c|}{$\begin{array}{l}\text { Secondary/high school } \\
\text { completed }\end{array}$} & 10.6 & 9.4 & 11.9 & 5.9 & 5.0 & 6.8 & 5.5 & 4.7 & 6.4 & 6.2 & 5.1 & 7.3 & 3.2 & 2.5 & 4.0 & 6.6 & 5.6 & 7.7 \\
\hline & \multicolumn{2}{|c|}{$\begin{array}{l}\text { College completed } \\
\text { or above }\end{array}$} & 9.0 & 7.5 & 10.6 & 3.9 & 2.9 & 4.9 & 3.1 & 1.9 & 4.3 & 3.4 & 2.2 & 4.5 & 3.5 & 2.6 & 4.4 & 3.9 & 2.9 & 4.9 \\
\hline & \multirow{2}{*}{$\begin{array}{l}\text { Slope index } \\
\text { of inequality }\end{array}$} & Model $1^{*}$ & 7.8 & 4.6 & 11.0 & 2.6 & 0.2 & 5.0 & 1.9 & -0.1 & 3.8 & 6.6 & 4.3 & 8.9 & -4.6 & -6.7 & -2.5 & 5.3 & 2.9 & 7.8 \\
\hline & & Model 2** & 3.8 & 0.2 & 7.5 & 1.2 & -1.7 & 4.1 & 1.3 & -0.8 & 3.4 & 4.5 & 1.7 & 7.3 & -1.8 & -3.4 & -0.1 & 3.9 & 1.0 & 6.8 \\
\hline
\end{tabular}

Model 1 is adjusted for country of residence and age.

** Model 2 is adjusted for country of residence, age, marital status, urban/rural area and wealth. 


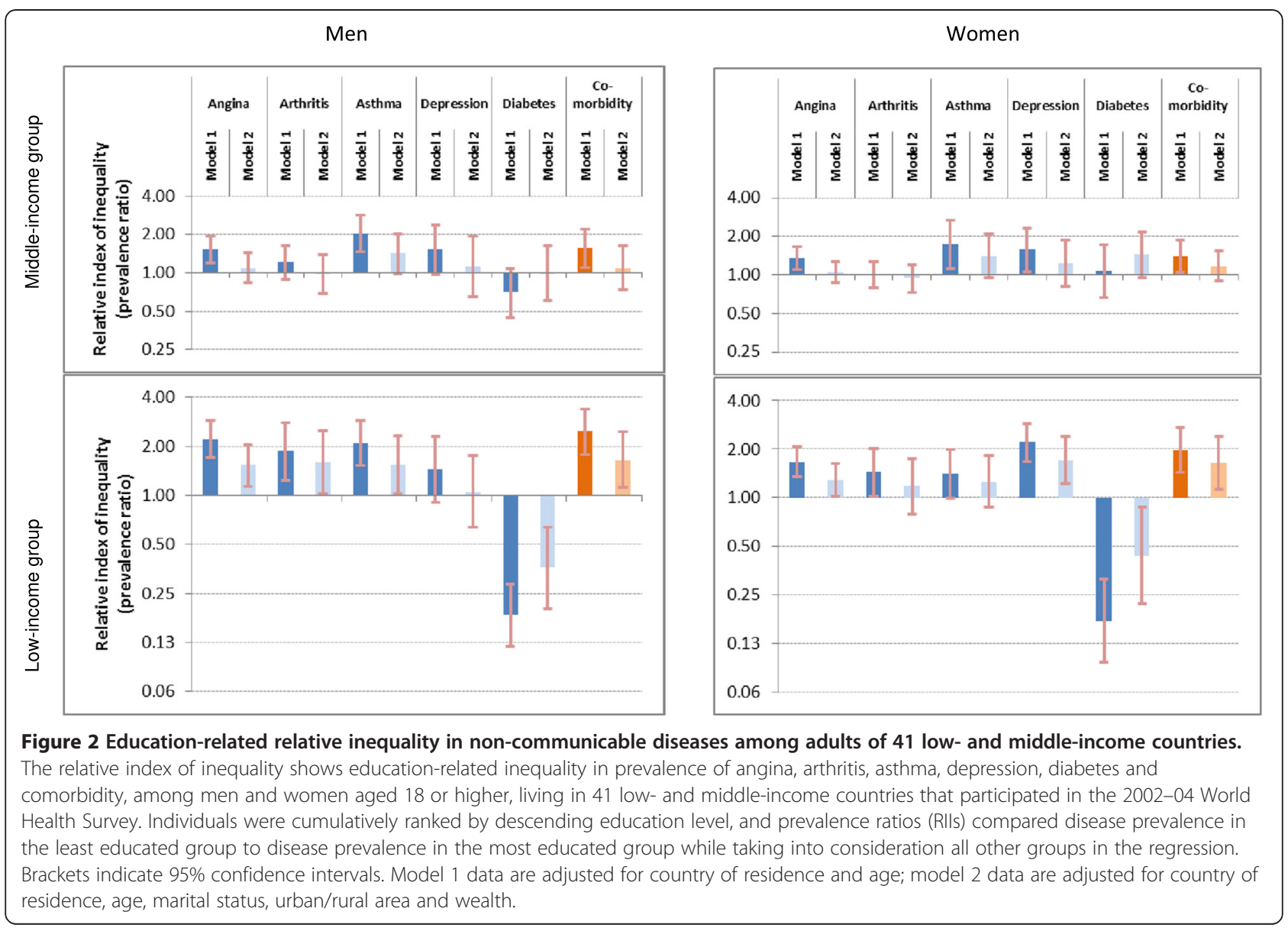

adolescents in India [67]. Nations at different levels of development may realize different stages of disease epidemiological transitions [67]. Noting methodological differences in determining diabetes prevalence, it is also possible that our findings may be subjected to bias stemming from a methodology issue whereby cases with a lower wealth or education were more likely to be underdiagnosed and therefore prevalence rates were underestimated. Populations in less-developed nations may have limited access to medical professionals [6], which could result in under-diagnosis of diabetes, particularly among populations of lower socio-economic status; for example, better educated individuals may be more aware of diabetes as a health condition. Alternatively, this finding may reflect a complex relationship between wealth, overweight, obesity, other risk factors (such as physical inactivity), and diabetes [68]. Ideally, future surveys may integrate objective indicators of disease, such as HbA1C diagnostic testing for diabetes [69].

Comorbidity significantly lowers quality of life, affecting physical, social and psychological well-being [70]. Our findings showed that comorbidity was more prevalent among the poor and less educated, in all sexincome groups. We reported overall comorbidity rates of up to $10 \%$, with even greater prevalence in some poorer wealth quintiles and least educated subpopulations. In a multinational study of high-income countries $30.2 \%$ of adults over 18 reported more than one chronic condition, although the study included seven diseases whereas ours included five [71]. Consistent with the present study, previous research has reported inverse associations between comorbidity and markers of socioeconomic status [30].

Like other studies, NCD prevalence tended to be higher in women than men [30], and the greatest burden was reported for a cardiovascular-related condition [3]. Overall, study NCDs tended to be more prevalent in the MIC group, with the exception of depression. That depression rates were higher in LICs than MICs was not expected. Previously, depression prevalence was reported to be lower in a less-developed setting, although cultural willingness to report depressive symptoms may bias outcomes [72]. According to epidemiological transition models, LICs may be expected to carry a lower-- albeit increasing-- burden of NCDs than higher-income country groups, as risk factors for infectious diseases are progressively replaced by risk factors for NCDs [73]. Projections for 2005-2030 forecasted a 10\% increase in 
the deaths due to chronic disease in LMICs (from $61 \%$ to $71 \%$ ) [74]. Monitoring trends in NCD prevalence in LMIC groups will help to characterize the nature of the modern epidemiological transition, and identify populations that are most at risk for NCDs.

\section{Strengths, limitations and implications}

Data for five NCDs were collected systematically in a large sample of LMICs that participated in the WHS, allowing for comparisons of standardized data across pooled data sets. Consistent diagnostic criteria in WHS data facilitated broad-scale analyses and comparisons across several countries, minimizing limitations associated with variable measurement tools and disease classifications. However, the use of pooled data from geographically- and culturally-diverse settings inevitably masks problems of comparability between countries [54]. Nine studies were excluded from analysis due to insufficient data or high item non-response rates. There is no reason to believe that the excluded countries would have changed the main findings on socioeconomic inequality in LMICs. The non-response was not selective, including countries of both low and middle income groups. We included a country variable in our multivariate analysis in order to control for any potential confounding effect of the individual countries. We did not aim to explore the interaction effects of our study's independent variables with each of the countries.

Wealth and education levels were determined nationally, and pooled across LICs and MICs. We acknowledge that patterns of wealth distribution vary between countries, however, quintile classification provided a widely accepted method to compare respondents based on relative wealth position within their country. Levels of education were standardized to be comparable across countries.

The use of symptom-based diagnoses for angina, arthritis, asthma and depression was a strength, as other methods that rely on medical charts or self-reported diagnoses may introduce biases related to health system access. Self-reported data could reflect systematic overor under-reporting, which may vary by socioeconomic status [75]. A tendency for under-reporting of symptoms by people with low levels of education [76] raises the possibility that our data may underestimate prevalence in low education classes, and show weaker-than-actual inverse associations. As a result, our data may underestimate true NCD rates in socioeconomic disadvantaged populations.

It is possible that a selection bias may have occurred in the sampling process, especially in countries with lower response rate, although we are not aware of evidence to suggest that this had occurred. The main reasons for household non-response included inability to locate the selected household, or household refusal to participate even before a roster could be obtained.

\section{Conclusions}

Action taken in the next 20 years will be critical in determining the outcome of the mounting NCD epidemic [77]. One of the major reasons for the relative failure of NCD advocacy is a lack of emphasis on social justice and inequality [78]. Delineating the impact of NCDs on poor and rich populations-- both between countryincome groupings and within countries --is an important precursor to NCD prevention and management efforts.

The focus of this study was to assess within-country socioeconomic distribution of five NCDs and comorbidity in LMICs. We reported disparities between subpopulations of different wealth- and education-levels, which varied according to the type of NCD. With the exception of diabetes, four NCDs and their comorbidity showed some evidence for unequal distribution in populations, to the detriment of those with lower wealth or education levels. Angina, asthma and comorbidity prevalence demonstrated the strongest inverse associations with wealth and education; arthritis and depression also reported inverse wealth and education associations in most cases. In LICs, diabetes prevalence was significantly positively associated with wealth and education.

Overall, our mixed findings substantiate the need for disaggregated research to delineate the impact of individual NCDs on various socioeconomic groups. High quality epidemiological evidence is a cornerstone of effective policy development, deployment and monitoring. The present study has shown that NCDs are not necessarily diseases of the wealthy, demonstrating unequal distribution across socioeconomic groups. Further investigation of the risk factors and root causes of socioeconomic inequality are warranted to formulate sustainable and effective approaches to prevent and manage NCDs among the poor and low educated.

\section{Additional files}

\footnotetext{
Additional file 1: Local review boards for study countries. Lists each study country and its corresponding local review board.

Additional file 2: Study sample size by country and sex, World Health Survey 2002-04. Displays the study sample size of men and women (aged 18 or higher) from 41 low- and middle-income countries that participated in the 2002-04 World Health Survey.

Additional file 3: Noncommunicable diseases non-response rates, by country and sex, World Health Survey 2002-04. Displays the nonresponse rates to World Health Survey individual questionnaires for each studied noncommunicable disease, grouped by sex and low- or middleincome country status. Data represent 41 low- and middle-income countries that participated in the 2002-04 World Health Survey.
}

Additional file 4: Crude prevalence (\%) of noncommunicable diseases among adults aged 18 or higher living in 41 low- and 
middle-income countries, World Health Survey 2002-04. Displays the crude prevalence rates (percentage) of each studied noncommunicable disease and comorbidity among adults (aged 18 or higher), grouped by sex and low- or middle-income country status. Data represent 41 lowand middle-income countries that participated in the 2002-04 World Health Survey.

Additional file 5: Wealth-related relative inequality in noncommunicable disease prevalence among adults aged 18 or higher living in $\mathbf{4 1}$ low- and middle-income countries, World Health Survey 2002-04. Displays the relative index of inequality and corresponding 95\% confidence interval for each studied noncommunicable disease and comorbidity among adults (aged 18 or higher), according to wealth quintile. Data are grouped by sex and lowor middle-income country status, and represent 41 low- and middleincome countries that participated in the 2002-04 World Health Survey. Model 1 data are adjusted for country of residence and age; model 2 data are adjusted for country of residence, age, marital status, urban/rural area and education.

Additional file 6: Crude prevalence (\%) of non-communicable diseases among adults aged 18 or higher living in 41 low- and middle-income countries, by wealth, World Health Survey 2002-04. Displays the crude prevalence rates (percentage) of each studied noncommunicable disease and comorbidity among adults (aged 18 or higher), according to wealth quintile. Data are grouped by sex and lowor middle-income country status, and represent 41 low- and middleincome countries that participated in the 2002-04 World Health Survey.

\section{Additional file 7: Education-related relative inequality in} noncommunicable disease prevalence among adults aged 18 or higher living in $\mathbf{4 1}$ low- and middle-income countries, World Health Survey 2002-04. Displays the relative index of inequality and corresponding 95\% confidence interval for each studied noncommunicable disease and comorbidity among adults (aged 18 or higher), according to education level. Data are grouped by sex and lowor middle-income country status, and represent 41 low- and middleincome countries that participated in the 2002-04 World Health Survey. Model 1 data are adjusted for country of residence and age; model 2 data are adjusted for country of residence, age, marital status, urban/rural area and wealth.

Additional file 8: Crude prevalence (\%) of noncommunicable diseases among adults aged 18 or higher living in 41 low- and middle-income countries, by education, World Health Survey 200204. Displays the crude prevalence rates (percentage) of each studied noncommunicable disease and comorbidity among adults (aged 18 or higher), according to education level. Data are grouped by sex and lowor middle-income country status, and represent 41 low- and middleincome countries that participated in the 2002-04 World Health Survey.

\section{Abbreviations}

LICs: Low-income countries; LMICs: Low- and middle-income countries; MICs: Middle-income countries; NCDs: Noncommunicable diseases; RII: Relative index of inequality; SII: Slope index of inequality; WHS: World Health Survey.

\section{Competing interests}

The authors declare that they have no competing interests.

\section{Authors' contributions}

$\mathrm{AH}$ designed the study. AH did the statistical analysis with inputs from EV. NB wrote the first draft with inputs from AH. AK, SH, SM, SC read the draft and provided critical comments. All authors read and approved the final draft.

\section{Acknowledgements}

The views expressed in this paper are those of the author(s) and do not necessarily represent the views or policies of the World Health Organization. This study was funded by World Health Organization as well as the United States of America, National Institutes of Health grant 1 R01 AG034479-01A1. The funders had no role in study design, data collection and analysis, decision to publish, or preparation of the manuscript.

\section{Author details}

'Department of Health Statistics and Information Systems, World Health Organization, Geneva, Switzerland. 'Department of Chronic Diseases and Health Promotion, World Health Organization, Geneva, Switzerland.

${ }^{3}$ Department of Epidemiology, Biostatistics \& Occupational Health, McGill University, Montreal, Canada. ${ }^{4}$ Department of Public Health, AMC, University of Amsterdam, Amsterdam, Netherlands.

Received: 12 December 2011 Accepted: 30 May 2012

Published: 22 June 2012

\section{References}

1. World Health Organization: 2008-2013 action plan for the global strategy for the prevention and control of noncommunicable diseases: prevent and control cardiovascular diseases, cancers, chronic respiratory diseases and diabetes. Geneva: World Health Organization; 2008.

2. Magnusson RS: Global health governance and the challenge of chronic non-communicable disease. J Law Med Ethics 2010, 38:490-507.

3. World Health Organization: World Health Organization: Global status report on noncommunicable diseases. Geneva: World Health Organization; 2011.

4. Moussavi S, Chatterji S, Verdes E, Tandon A, Patel V, Ustun B: Depression, chronic diseases, and decrements in health: results from the World Health Surveys. Lancet 2007, 370:851-858

5. Gabriel SE: The epidemiology of rheumatoid arthritis. Rheum Dis Clin North Am 2001, 27:269-281.

6. Leeder S, Raymond S, Greenberg H, Liu H, Esson K: A race against time: the challenge of cardiovascular disease in developing economies. New York: The Center for Global Health and Economic Development; 2004

7. Kaplan GA, Keil JE: Socioeconomic factors and cardiovascular disease: a review of the literature. Circulation 1993, 88:1973-1998.

8. Theme-Filha MM, Szwarcwald CL, Souza-Junior PR: Socio-demographic characteristics, treatment coverage, and self-rated health of individuals who reported six chronic diseases in Brazil, 2003. Cad Saude Publica 2005, 21(Suppl):43-53

9. Dalstra JA, Kunst AE, Borrell C, Breeze E, Cambois E, Costa G, Geurts JJ, Lahelma E, Van OH, Rasmussen NK, Regidor E, Spadea T, Mackenbach JP: Socioeconomic differences in the prevalence of common chronic diseases: an overview of eight European countries. Int J Epidemiol 2005, 34:316-326.

10. Liu L, Ma J, Yin X, Kelepouris E, Eisen HJ: Global variability in angina pectoris and its association with body mass index and poverty. Am J Cardiol 2011, 107:655-661.

11. Lee G, Carrington M: Tackling heart disease and poverty. Nurs Health Sci 2007, 9:290-294.

12. Callahan LF: Social epidemiology and rheumatic disease. Curr Opin Rheumatol 2003, 15:110-115.

13. Callahan LF, Shreffler J, Mielenz T, Schoster B, Kaufman JS, Xiao C, Randolph R, Sloane PD: Arthritis in the family practice setting: associations with education and community poverty. Arthritis Rheum 2008, 59:1002-1008.

14. Bengtsson C, Nordmark B, Klareskog L, Lundberg I, Alfredsson L: Socioeconomic status and the risk of developing rheumatoid arthritis: results from the Swedish EIRA study. Ann Rheum Dis 2005, 64:1588-1594.

15. Busija L, Hollingsworth B, Buchbinder R, Osborne RH: Role of age, sex, and obesity in the higher prevalence of arthritis among lower socioeconomic groups: a population-based survey. Arthritis Rheum 2007, 57:553-561.

16. Canizares M, Power JD, Perruccio AV, Badley EM: Association of regional $\mathrm{racial} /$ cultural context and socioeconomic status with arthritis in the population: a multilevel analysis. Arthritis Rheum 2008, 59:399-407.

17. Callahan LF, Shreffler J, Siaton BC, Helmick CG, Schoster B, Schwartz TA, Chen JC, Renner JB, Jordan JM: Limited educational attainment and radiographic and symptomatic knee osteoarthritis: a cross-sectional analysis using data from the Johnston County (North Carolina) Osteoarthritis Project. Arthritis Res Ther 2010, 12:R46.

18. Stewart AW, Mitchell EA, Pearce N, Strachan DP, Weiland SK: The relationship of per capita gross national product to the prevalence of symptoms of asthma and other atopic diseases in children (ISAAC). Int J Epidemiol 2001, 30:173-179.

19. Sembajwe G, Cifuentes M, Tak SW, Kriebel D, Gore R, Punnett L: National income, self-reported wheezing and asthma diagnosis from the World Health Survey. Eur Respir J 2010, 35:279-286. 
20. Volmer T: The socio-economics of asthma. Pulm Pharmacol Ther 2001, 14:55-60.

21. Lai CK, Beasley R, Crane J, Foliaki S, Shah J, Weiland S: Global variation in the prevalence and severity of asthma symptoms: phase three of the International Study of Asthma and Allergies in Childhood (ISAAC). Thorax 2009, 64:476-483.

22. Littlejohns P, Macdonald LD: The relationship between severe asthma and social class. Respir Med 1993, 87:139-143.

23. Patel V: Cultural factors and international epidemiology. Br Med Bull 2001, 57:33-45.

24. Lorant V, Deliege D, Eaton W, Robert A, Philippot P, Ansseau M: Socioeconomic inequalities in depression: a meta-analysis. Am J Epidemiol 2003, 157:98-112.

25. Connolly V, Unwin N, Sherriff P, Bilous R, Kelly W: Diabetes prevalence and socioeconomic status: a population based study showing increased prevalence of type 2 diabetes mellitus in deprived areas. J Epidemiol Community Health 2000, 5:4173-177.

26. Glover JD, Hetzel DM, Tennant SK: The socioeconomic gradient and chronic illness and associated risk factors in Australia. Aust New Zealand Health Policy 2004, 1:8.

27. Goyal A, Bhatt DL, Steg PG, Gersh BJ, Alberts MJ, Ohman EM, Corbalan R, Eagle KA, Gaxiola E, Gao R, Goto S, D'Agostino RB, Califf RM, Smith SC Jr, Wilson PW: Attained educational level and incident atherothrombotic events in low- and middle-income compared with high-income countries. Circulation 2010, 122:1167-1175.

28. Fleischer NL, Diez Roux AV, Alazraqui M, Spinelli H: Social patterning of chronic disease risk factors in a Latin American city. J Urban Health 2008, 85:923-937.

29. Minh HV, Huong DL, Giang KB: Self-reported chronic diseases and associated sociodemographic status and lifestyle risk factors among rural Vietnamese adults. Scand J Public Health 2008, 36:629-634.

30. Van MH, Ng N, Juvekar S, Razzaque A, Ashraf A, Hadi A, Soonthornthada K, Kanungsukkasem U, Bich TH, Byass P: Self-reported prevalence of chronic diseases and their relation to selected sociodemographic variables: a study in INDEPTH Asian sites, 2005. Prev Chronic Dis 2008, 5:A86.

31. Meetoo D: Chronic diseases: the silent global epidemic. Br J Nurs 2008, 17:1320-1325

32. Alwan A, Maclean DR, Riley LM, DESpaignet ET, Mathers CD, Stevens GA, Bettcher D: Monitoring and surveillance of chronic non-communicable diseases: progress and capacity in high-burden countries. Lancet 2010, 376:1861-1868.

33. Yach D, Kellogg M, Voute J: Chronic diseases: an increasing challenge in developing countries. Trans R Soc Trop Med Hyg 2005, 99:321-324.

34. In A prioritized research agenda for prevention and control of noncommunicable diseases. Edited by Mendis S, Alwan A. Geneva: World Health Organization; 2011:11.

35. Wissow LS: Diabetes, poverty, and Latin America. Patient Educ Couns 2006, 61:169-170

36. World Health Survey Instruments and Related Documents. 2011. http:// www who int/healthinfo/survey/instruments/en/index htm.

37. Ustun TB, Chatterii S, Mechbal A, Murray CJL: The World Health Surveys. In In Health systems performance assessment debates, methods and empiricisms. Edited by Murray C, Evans D. Geneva: World Health Organization; 2003:97-808.

38. United Nations Statistics Division. 2011. http://unstats un org/unsd/default $\mathrm{htm}$.

39. Hosseinpoor AR, Stewart Williams J, Amin A, Araujo DC I, Beard J, Boerma T, Kowal P, Naidoo N, Chatterji S: Social determinants of self-reported health in women and men: understanding the role of gender in population health. PloS One 2012, 7:e34799.

40. World Bank: World Development Report 2003: Sustainable development in a dynamic world. Transforming institutions, growth and quality of life. Washington DC: World Bank; 2002.

41. Rose G: The diagnosis of ischaemic heart pain and intermittent claudication in field surveys. Bull World Health Organ 1962, 27:645-658.

42. Veenhof C, Bijlsma JW, van den Ende CH, van Dijk GM, Pisters MF, Dekker J: Psychometric evaluation of osteoarthritis questionnaires: a systematic review of the literature. Arthritis Rheum 2006, 55:480-492.

43. Pekkanen J, Pearce N: Defining asthma in epidemiological studies. Eur Respir J 1999, 14:951-957.
44. Torén K, Brisman J, Järvholm B: Asthma and asthma-like symptoms in adults assessed by questionnaires. Chest 1993, 104:600-608.

45. World Health Organization: The ICD-10 classification of mental and behavioural disorders: diagnosis criteria for research (DCR-10). Geneva: World Health Organization; 1993.

46. Kessler RC, Ustun TB: The World Mental Health (WMH) Survey Initiative Version of the World Health Organization (WHO) Composite International Diagnostic Interview (CIDI). Int J Methods Psychiatr Res 2004 13:93-121.

47. Armitage P, Berry G, Matthews JNS: Statistical methods in epidemiology. In Statistical methods in medical research. 4th edition. Malden: Blackwell Science Ltd; 2002:648-716.

48. Gakidou E, Oza S, Vidal FC, Li AY, Lee DK, Sousa A, Hogan MC, Vander HS, Ezzati M: Improving child survival through environmental and nutritional interventions: the importance of targeting interventions toward the poor. JAMA 2007, 298:1876-1887.

49. Ferguson BD, Tandon A, Gakidou E, Murray CJ: Estimating permanent income using asset and indicator variables. In Health systems performance assessment: debates, methods and empiricism. Edited by Murray CJL, Evans DB. Geneva: World Health Organization; 2003:747-760.

50. Hosseinpoor AR, Mohammad K, Majdzadeh R, Naghavi M, Abolhassani F, Sousa A, Speybroeck N, Jamshidi HR, Vega J: Socioeconomic inequality in infant mortality in Iran and across its provinces. Bull World Health Organ 2005, 83:837-844.

51. Ahmad O, Boschi-Pint C, Lopez A, Murray C, Lozano R, Inoue M: Age standardization of rates: a new WHO standard. Geneva: World Health Organization; 2001

52. Harper S, Lynch J: Measuring inequalities in health. In Methods in social epidemiology. Edited by Oakes J, Kaufman JS. San Francisco: Jossey-BasS; 2006:134-168

53. Barros AJ, Hirakata VN: Alternatives for logistic regression in crosssectional studies: an empirical comparison of models that directly estimate the prevalence ratio. BMC Med Res Methodol 2003, 3:21.

54. Mackenbach JP, Stirbu I, Roskam AJ, Schaap MM, Menvielle G, Leinsalu M, Kunst AE: Socioeconomic inequalities in health in 22 European countries. N Engl J Med 2008, 358:2468-2481.

55. Royston P: Multiple imputation of missing values: update. The Stata Journal 2005, 5:188-201.

56. Rose G, Marmot MG: Social class and coronary heart disease. Br Heart J 1981, 45:13-19.

57. Eagan TM, Gulsvik A, Eide GE, Bakke PS: The effect of educational level on the incidence of asthma and respiratory symptoms. Respir Med 2004, 98:730-736.

58. Li X, Sundquist J, Sundquist K: Socioeconomic and occupational groups and risk of asthma in Sweden. Occup Med (Lond) 2008, 58:161-168

59. Yunming L, Changsheng C, Haibo T, Wenjun C, Shanhong F, Yan M, Yongyong $X$, Qianzhen $\mathrm{H}$ : Prevalence and risk factors for depression in older people in Xi'an China: a community-based study. Int I Geriatr Psychiatry 2012, 27:31-39.

60. Muhammad Gadit AA, Mugford G: Prevalence of depression among households in three capital cities of Pakistan: need to revise the mental health policy. PLoS One 2007, 2:e209.

61. Lee J: Pathways from Education to Depression. J Cross Cult Gerontol 2011 26:121-135.

62. Hamad R, Fernald LC, Karlan DS, Zinman J: Social and economic correlates of depressive symptoms and perceived stress in South African adults. J Epidemiol Community Health 2008, 62:538-544.

63. Commission on Social Determinants of Health: Closing the gap in a generation: health equity through action on the social determinants of health: final report of the commission on social determinants of health. Geneva: World Health Organization; 2008.

64. World Health Organization: Preventing chronic diseases: a vital investment: WHO global report. Geneva: World Health Organization; 2005.

65. Unwin N, Alberti KG: Chronic non-communicable diseases. Ann Trop Med Parasitol 2006, 100:455-464.

66. Acosta D, Rottbeck R, Rodriguez JG, Gonzalez LM, Almanzar MR, Minaya SN, Ortiz MC, Ferri CP, Prince MJ: The prevalence and social patterning of chronic diseases among older people in a population undergoing health transition. A 10/66 Group cross-sectional population-based survey in the Dominican Republic. BMC Public Health 2010, 10:344. 
67. Singh R, Bhansali A, Sialy R, Aggarwal A: Prevalence of metabolic syndrome in adolescents from a north Indian population. Diabet Med 2007, 24:195-199.

68. Reddy KS: Cardiovascular diseases in the developing countries: dimensions, determinants, dynamics and directions for public health action. Public Health Nutr 2002, 5:231-237.

69. WHO Study on Global AGEing and Adult Health (SAGE). 2011. http://www who int/healthinfo/systems/sage/en/index html.

70. Fortin M, Lapointe L, Hudon C, Vanasse A, Ntetu AL, Maltais D: Multimorbidity and quality of life in primary care: a systematic review. Health Qual Life Outcomes 2004, 2:51.

71. Alonso J, Ferrer M, Gandek B, Ware JE Jr, Aaronson NK, Mosconi P, Rasmussen NK, Bullinger M, Fukuhara S, Kaasa S, Leplege A: Health-related quality of life associated with chronic conditions in eight countries: results from the International Quality of Life Assessment (IQOLA) Project. Qual Life Res 2004, 13:283-298.

72. Ball HA, Siribaddana SH, Kovas Y, Glozier N, McGuffin P, Sumathipala A, Hotopf M: Epidemiology and symptomatology of depression in Sri Lanka: a cross-sectional population-based survey in Colombo District. J Affect Disord 2010, 123:188-196.

73. World Health Organization: Global health risks: mortality and burden of disease attributable to selected major risks. Geneva: World Health Organization; 2009

74. Abegunde DO, Mathers CD, Adam T, Ortegon M, Strong K: The burden and costs of chronic diseases in low-income and middle-income countries. Lancet 2007, 370:1929-1938.

75. Kehoe R, Wu SY, Leske MC, Chylack LT Jr: Comparing self-reported and physician-reported medical history. Am J Epidemiol 1994, 139:813-818.

76. Mackenbach JP, Looman CW, van der Meer JB: Differences in the misreporting of chronic conditions, by level of education: the effect on inequalities in prevalence rates. Am J Public Health 1996, 86:706-711.

77. Greenberg H, Raymond SU, Leeder SR: Cardiovascular disease and global health: threat and opportunity. Health Aff (Millwood) 2005, Web Suppl:W5 31-W5 41

78. Stuckler D, Basu S, McKee M: Commentary: UN high level meeting on noncommunicable diseases: an opportunity for whom? In BMJ 2011, 343: d5336.

doi:10.1186/1471-2458-12-474

Cite this article as: Hosseinpoor et al: Socioeconomic inequality in the prevalence of noncommunicable diseases in low- and middle-income countries: Results from the World Health Survey. BMC Public Health 2012 12:474.

\section{Submit your next manuscript to BioMed Central and take full advantage of:}

- Convenient online submission

- Thorough peer review

- No space constraints or color figure charges

- Immediate publication on acceptance

- Inclusion in PubMed, CAS, Scopus and Google Scholar

- Research which is freely available for redistribution 